\title{
First principles molecular dynamics study of nitrogen vacancy complexes in boronitrene
}

\author{
A M Ukpong ${ }^{1, *}$, and N Chetty ${ }^{1,2}$ \\ ${ }^{1}$ Department of Physics, University of Pretoria, Hatfield 0002, South Africa \\ ${ }^{2}$ National Institute for Theoretical Physics, Johannesburg 2000, South Africa \\ *E-mail: aniekan.ukpong@up.ac.za
}

\begin{abstract}
We present the results of first principles molecular-dynamics simulations of nitrogen vacancy complexes in monolayer hexagonal boron nitride. The threshold for local structure reconstruction is found to be sensitive to the presence of a substitutional carbon impurity. We show that an activated nitrogen dynamics triggers the annihilation of defects in the layer through formation of Stone-Wales-type structures. The lowest energy state of nitrogen vacancy complexes is negatively-charged and spin-polarised. Using the divacancy complex, we show that their formation induces spontaneous magnetic moments, which is tuneable by electron or hole injection. The Fermi level $s$-resonant defect state is identified as a unique signature of the ground state of the divacancy complex. Due to their ability to enhance the structural cohesion, only the di-vacancy and the nitrogen vacancy carbon-antisite complexes are able to suppress the Fermi level resonant defect state to open a gap between the conduction and valence bands.
\end{abstract}

PACS numbers: $71.15 . \mathrm{Nc}, 71.55 . \mathrm{Ht}, 73.22 . \mathrm{Pr}$

\section{Introduction}

Boronitrene, the freestanding monolayer of hexagonal boron nitride (h-BN) [1-3] has attracted considerable interests in recent times as the boron nitride analogue of graphene [4-6]. Because h-BN has wide band gap $(\sim 5-7 \mathrm{eV})$ and no lattice mismatch with graphene, thin h-BN layers are potentially useful as thin top-dielectric to gate graphene [7,8]. Its low chemical activity also makes it attractive as an inert insulating membrane for graphene isolation [8-10]. When used as the buffer layer in graphene heterojunctions, it inhibits the effects of interface disorder and improves the electronic quality of the electronic device [11]. Its emergence as a tunnelling barrier (or spacer) in narrow-spaced quantumwell heterojunctions [12] underscores the need to identify and characterize the effects of the band edge and mid-gap defect states on the optical [13-15] and magnetic [16-18] properties. Our previous studies have shown that the substitutional carbon impurity on boron $\left(C_{B}\right)$ and nitrogen $\left(C_{N}\right)$ sites are the most stable defects in monolayer h-BN [19]. Because knock-on electron damage can create vacancies on B, $\mathrm{N}$ or both sublattices, we consider the binding of vacancies with the substitutional carbon impurities.

Following the discovery of spontaneous magnetism in pure carbon systems [20-24], several attempts have been made to synthesis hybrid BCN nanostructures [25-30]. Such hybrid nanostructures - in particular, graphene flakes embedded in the h-BN monolayer - possess tuneable magnetic moments [31,32], among many other interesting electronic properties. Negatively charged h-BN nanostructures have been obtained by postsynthetic doping with substitutional carbon impurity by in 
situ electron beam irradiation inside the transmission electron microscope [33,34]. Irradiation with charged particles changes the layer from an insulator to a conductor under zero bias voltage. This offers a new way of controlling the electronic properties of $\mathrm{h}-\mathrm{BN}$ nanostructures by tuning its charge and spin states [35]. The electronic properties of point defects in monolayer h-BN have been studied in several ab initio methods [36-43], but few of these studies consider magnetic properties [16-18], and so far, none has considered the effects of finite temperature on these properties. This paper focuses on the finite temperature properties of the complexes that form when point defects bind with nitrogen vacancy on the nearest neighbour position. We employ spin-polarised calculations to explore the charged states of nitrogen vacancy complexes for spontaneous magnetism under conditions of finite temperature. We show that activated migration of nitrogen leads to the annihilation of defects in monolayer h-BN, and identify the threshold for reconstruction of the local structure.

\section{Computational approach}

First principles molecular dynamics simulations were performed using the Vienna ab initio simulation package (VASP) [44-47] as implemented in the MedeA suite [48]. The exchange-correlation potential was described in the generalized gradient approximation as parameterized by Perdew, Burke and Ernzerhof (GGA-PBE) [49]. Plane waves were included up to $500 \mathrm{eV}$ for the kinetic energy expansion, and the limit of $10^{-7} \mathrm{eV}$ was set for total energy convergence. The projector augmented wave potentials $[50,51]$ were used to describe ionic interactions, and the Monkhorst-Pack scheme was used to sample the Brillouin zone [52]. The h-BN monolayer was modelled using a $5 \times 5$ supercell, and a vacuum region of height $15 \AA$ was used to minimize spurious interlayer interactions. The hexagonal symmetry of the layer was maintained for lattice relaxations at $300 \mathrm{~K}$.

Because the reaction kinetics involved in experimental growth of $h-B N$ layers requires the layer to form from hyperthermal ions, we adopt the melt-quenching procedure in our simulations. The simulations start with well-equilibrated liquid structure, obtained by heating h-BN structures to 3500 $\mathrm{K}$ in the canonical NVT ensemble. This scheme ensures that the liquid forms at a temperature higher than the low-pressure $(400 \pm 20 \mathrm{~Pa})$ melting point $(3400 \pm 20 \mathrm{~K})$ of bulk hexagonal boron nitride [53]. The 'high-temperature' liquid was equilibrated for 240 ps before slow-cooling to $300 \mathrm{~K}$ at $1.5 \times 10^{-10}$ $\mathrm{K} / \mathrm{sec}$. The slow cooling ensures recrystallization to the hexagonal structure at the same density. However, we note that a direct equilibration of the h-BN structure yields similar results. Upon forming nitrogen vacancy complexes, the total energy was calculated in both non-magnetic and spin-polarized charged states. Total energy was converged with respect to supercell size using the same tests reported in Ref. [19]. Because system size only affects nucleation time for formation of crystallites from a supercooled liquid phase [54], we assume that supercell size has trivial effects on the properties of the resulting h-BN layers at finite temperature. The layers were equilibrated at $300 \mathrm{~K}$, in each case, in NVE simulations over 50 ps until the average force on each atom is less than $0.002 \mathrm{eV} / \AA$.

The defect formation energy was calculated using the Zhang-Northrup formalism [55], in which the number of atoms $n_{i}$, and chemical potential $\mu_{i}$, of the interacting species determine the formation energy of a neutral defect. In cases where the defect is charged, the defect formation energy $E_{f}^{q}$ is also determined by the electron chemical potential $\mu_{e}$ measured relative to the valence band maximum $\varepsilon_{v}$ or the Fermi level. The formation energy of a defect in charge state $q$ was calculated as $E_{f}^{q}(D)=E_{\text {total }}(D)+q\left(\mu_{e}+\varepsilon_{v}\right)-\sum_{i} n_{i} \mu_{i}$, where $E_{\text {total }}(D)$ is the total energy of a supercell containing defect $D$. The third term denotes the Gibbs free energy due to formation of $n_{i}$ species of type $i$ with chemical potential $\mu_{i}$ from B, N and $\mathrm{C}$ atoms, and bonded B-N pairs. Since the atomic chemical potentials are fixed by the condition $\mu_{\mathrm{B}}+\mu_{\mathrm{N}}=\mu_{\mathrm{BN}}$ at thermodynamic equilibrium, it follows that the upper bounds for $\mu_{\mathrm{B}}$ and $\mu_{\mathrm{N}}$ correspond to the precipitation limits for forming metallic boron $(\alpha-\mathrm{B})$ and molecular nitrogen $\left(\mathrm{N}_{2}\right)$, in each case. The chemical potentials are constrained by the 
conditions $\mu_{\mathrm{B}} \leq \mu_{\mathrm{B}}^{\text {bulk }}$ and $\mu_{\mathrm{N}} \leq \mu_{\mathrm{N}}^{\text {bulk }}$ so that the formation energy of the defective monolayers vary in the interval, $-\Delta H_{f} \leq \Delta \mu \leq \Delta H_{f}$, fixed by the heat of formation $\Delta H_{f}$ of the pristine h-BN monolayer. We calculated $\Delta H_{f}$ using $\Delta H_{f}=-\left(\mu_{\mathrm{BN}}-\mu_{\mathrm{B}}^{\text {bulk }}-\mu_{\mathrm{N}}^{\text {bulk }}\right)$, and obtained the value of $2.58 \mathrm{eV}$ [19]. Hence, $E_{f}^{q}(D)$ varies in the interval $-2.58 \mathrm{eV} \leq \Delta \mu \leq 2.58 \mathrm{eV}$, where the lower (i.e. $\Delta \mu=-\Delta H_{f}$ ) and upper (i. e. $\Delta \mu=+\Delta H_{f}$ ) bounds of the interval corresponds to nitrogen-rich and boron-rich growth conditions, respectively.

\section{Results and discussion}

\subsection{Finite temperature properties}

At $300 \mathrm{~K}$, the pristine $\mathrm{h}-\mathrm{BN}$ monolayer shows a mean outward relaxation of $\sim 0.1 \%$ in $\mathrm{B}-\mathrm{N}$ bond length relative to the optimised bond length of $1.451 \AA$ at $0 \mathrm{~K}$. The defect-free layer is no longer atomically flat at $300 \mathrm{~K}$. The out-of-plane bond relaxations cause them to stretch, resulting in a maximum buckling of $0.60 \AA$ along the $c$-axis. These distortions are minimal because entropic contribution from thermal broadening at $300 \mathrm{~K}$ is only $\sim 38 \mathrm{meV}$ higher than the free energy at $0 \mathrm{~K}$. We therefore conclude that the effect of finite temperature does not change the average bond angle from $120^{\circ}$. Because we have constrained our analysis to vacancy complexes that form on nearest neighbour positions only [19], we consider the cases where a nitrogen vacancy $\left(\mathrm{V}_{\mathrm{N}}\right)$ binds to either the vacancy on the boron site $\left(\mathrm{V}_{\mathrm{B}}\right)$, a boron atom which occupies the nitrogen site $\left(\mathrm{B}_{\mathrm{N}}\right)$, or to an impurity carbon atom which occupies the boron site $\left(\mathrm{C}_{\mathrm{B}}\right)$. Fig. 1 shows plane-averaged difference charge density superimposed on the local structure of the nitrogen vacancy complexes. The presence of defects causes non-uniform charge density redistribution along bond axes and hollow sites because of variations in bond lengths. This shows that cohesion in the layer arises from the flexibility of the inplane B-N bonds and the changes in bond lengths indicate that stability arises from puckering [56].
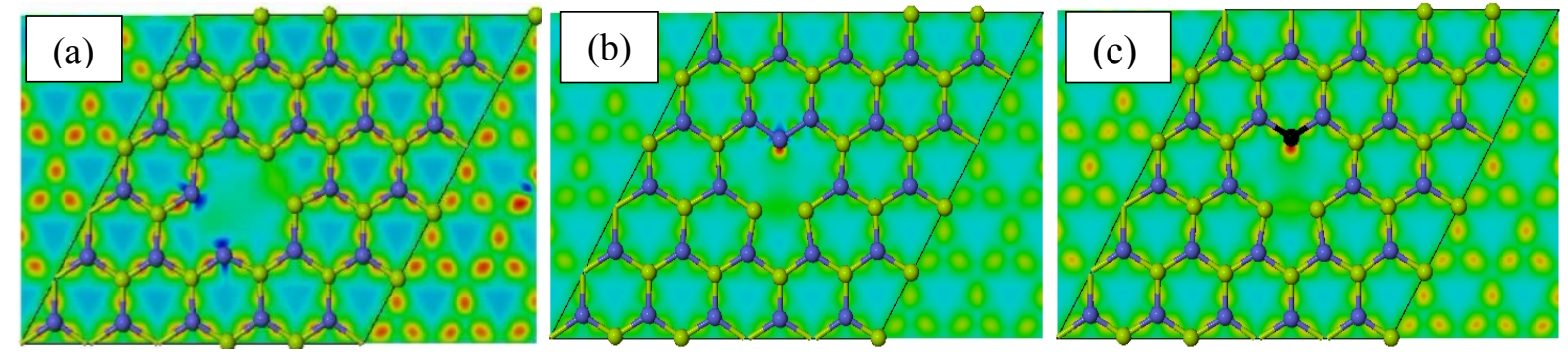

Figure 1. Distribution of difference charges in: $V_{N}+V_{B}(a) ; V_{N}+N_{B}(c)$; and the $V_{N}+C_{B}$ complexes.

Table I shows the cohesive energy of neutral defects from spin polarised calculations. The cohesive energy difference between a given defective and pristine layer was also calculated as $\delta=E_{c o h}(D)-E_{c o h}\left(\mathrm{~B}_{25} \mathrm{~N}_{25}\right)$. Mosuang and Lowther [37] first defined $\delta$ as the cohesive energy difference between bulk c-BN and h-BN that contain the same defect, in order to capture the role of point defects in the transition from cubic-to-hexagonal order. The cohesive energy is sensitive to stoichiometry because enhanced cohesion is observed in the stoichiometric $V_{N}+C_{B}$ complex $(-1.57 \mathrm{eV})$ compared to the nonstoichiometric $\mathrm{V}_{\mathrm{N}}+\mathrm{N}_{\mathrm{B}}$ complex $(-0.32 \mathrm{eV})$. Though $\delta$ is insensitive to the $\mathrm{B}, \mathrm{N}$ and $\mathrm{C}$ atomic energies used to calculate the cohesive energy, the evaluation of $\delta$ relative to the pristine h-BN permits its use to capture the role of defects on local bonding structure and cohesion.

The relaxation energies are negative, and charged defects also show similar trends in relaxation and cohesive energies. This shows the layer stability on the potential energy surface. The energy required to relax the $V_{N}+V_{B}$ structure is low compared to $V_{N}+C_{B}$ complex. This justifies why the magnitude of the re-distributed charge density along the B-N bonds [see Fig. 1(c)] is higher in $\mathrm{V}_{B}+C_{N}$ complex, on the same scale, compared to the $\mathrm{V}_{\mathrm{N}}+\mathrm{V}_{\mathrm{B}}$ complex. Local distortions in the charge density 
due to the presence of carbon in $\mathrm{V}_{\mathrm{N}}+\mathrm{C}_{\mathrm{B}}$ is therefore substantial along the N-C-N bonds to warrant the outward relaxation of the B-N bond length, and the significantly enhanced cohesive energy (see Table 1). The large difference in relaxation energy between $V_{N}+C_{B}$ and $V_{N}+N_{B}$ complexes alludes to the number of symmetry operations necessary to re-distribute the charge density compared to the $V_{N}+V_{B}$ complex. Thus, we conclude that the presence of substitutional carbon impurity has non-trivial effects on the properties of the layer.

The total energy of nitrogen vacancy complexes in spin and charge states are shown in Table 2 as offsets relative to the most stable configuration. Neutral and positively charged states of the $V_{N}+V_{B}$ complex are degenerate because their total energies are unchanged from non-magnetic and spin polarisation calculations. In stoichiometric complexes, all electrons have paired spins and zero net magnetic moment in the neutral state. The $\mathrm{V}_{\mathrm{N}}+\mathrm{V}_{\mathrm{B}}$ and $\mathrm{V}_{\mathrm{N}}+\mathrm{C}_{\mathrm{B}}$ complex are least stable in the positively charged state, and spin polarisation has no effect on its stability. The same argument is also valid for the $\mathrm{V}_{\mathrm{N}}+\mathrm{N}_{\mathrm{B}}$ complex showing that charge and spin states are non-trivial to defect stability. Table 2 shows that all negatively charged spin polarised complexes give the most stable configurations. These findings are consistent with the postsynthetic doping of h-BN layers with substitutional carbon impurity by electron irradiation [32-34], and show that layer stability is sensitive to the net electron density as electron rich layers are more stable than electron deficient ones.

Table 1. Relaxation and cohesive energies of hexagonal boron nitride monolayers containing nitrogen vacancy complexes.

\begin{tabular}{cccc}
\hline \hline Monolayer & $\delta(\mathrm{eV})$ & \multicolumn{2}{c}{ Energy } \\
\cline { 3 - 4 } & & $\begin{array}{c}\text { Relaxation } \\
(\mathrm{eV})\end{array}$ & $\begin{array}{c}\text { Cohesive } \\
(\mathrm{eV} / \text { atom })\end{array}$ \\
\hline Pristine & 0.00 & 0.00 & -0.40 \\
$\mathrm{~V}_{\mathrm{N}}+\mathrm{C}_{\mathrm{B}}$ & -1.17 & -0.96 & -1.57 \\
$\mathrm{~V}_{\mathrm{N}}+\mathrm{N}_{\mathrm{B}}$ & 0.08 & -1.31 & -0.32 \\
$\mathrm{~V}_{\mathrm{N}}+\mathrm{V}_{\mathrm{B}}$ & -0.07 & -0.81 & -0.47 \\
\hline \hline
\end{tabular}

Table 2. Offsets in total energy of nitrogen vacancy complexes in non-magnetic and spin-polarised charged states relative to their corresponding lowest energy state.

\begin{tabular}{ccccc}
\hline \hline & & \multicolumn{3}{c}{ Total energy difference $(\mathrm{eV})$} \\
\cline { 3 - 5 } Complex & Magnetism & $-e$ & 0 & $+e$ \\
\hline $\mathrm{V}_{\mathrm{N}}+\mathrm{V}_{\mathrm{B}}$ & & 0.07 & 2.53 & 6.98 \\
$\mathrm{~V}_{\mathrm{N}}+\mathrm{C}_{\mathrm{B}}$ & \multirow{2}{*}{ No spin } & 0.99 & 1.40 & 4.08 \\
$\mathrm{~V}_{\mathrm{N}}+\mathrm{N}_{\mathrm{B}}$ & & 0.00 & 0.71 & 3.77 \\
\hline $\mathrm{V}_{\mathrm{N}}+\mathrm{V}_{\mathrm{B}}$ & Spin & 0.00 & 2.53 & 6.98 \\
$\mathrm{~V}_{\mathrm{N}}+\mathrm{C}_{\mathrm{B}}$ & polarized & 0.00 & 1.40 & 3.99 \\
$\mathrm{~V}_{\mathrm{N}}+\mathrm{N}_{\mathrm{B}}$ & & 0.00 & 0.70 & 3.77 \\
\hline \hline
\end{tabular}

In Figs. 2(a)-(c), we present the formation energies of charged $\mathrm{V}_{\mathrm{N}}$-complexes as a function of the electron chemical potential in the N-rich condition. All the positively charged complexes have positive formation energies while the negatively charged complexes have negative formation energies. The positive formations energies indicate that formation of positively charged $\mathrm{V}_{\mathrm{N}}$-complexes in h-BN is not favourable. The magnitude of the positive formation energy increases continuously as the magnitude of the positive charge on the complexes increases. The magnitude of the formation energy of negatively charged complexes shows a decreasing trend as magnitude of the negative charges increases. For negatively charged complexes, there is a continuous decrease in $E_{f}$ as $\mu_{e}$ increases. These show that as more electrons are supplied to the nitrogen vacancy complexes, the energy penalty 
necessary for their formation decreases steadily. For the charged $\mathrm{V}_{\mathrm{N}}+\mathrm{C}_{\mathrm{B}}$ complex, $E_{f}$ shows only minimal variations with $\mu_{e}$. The most significant changes in $E_{f}$ are observed for transitions from $0 \rightarrow$ +1 and $0 \rightarrow-1$ charge states, indicating that substitutional carbon impurity is nontrivial in $V_{\mathrm{N}}$ defect. These results can be understood from the viewpoint of the minimisation of electronic perturbations in the presence of defects. When carbon replaces a boron atom in $V_{N}$ to form $V_{N}+C_{B}$ in the neutral state, one extra electron is added to the system. When the complex is positively charged - that is if one electron is removed to give the +1 charge state - the effect of the carbon substitution is effectively reversed, and the electronic structure resembles that of the neutral $\mathrm{V}_{\mathrm{N}}$. These trends are consistent with the dependence of $E_{f}$ on $\mu_{e}$ for charged carbon substitutional defects in h-BN [35].
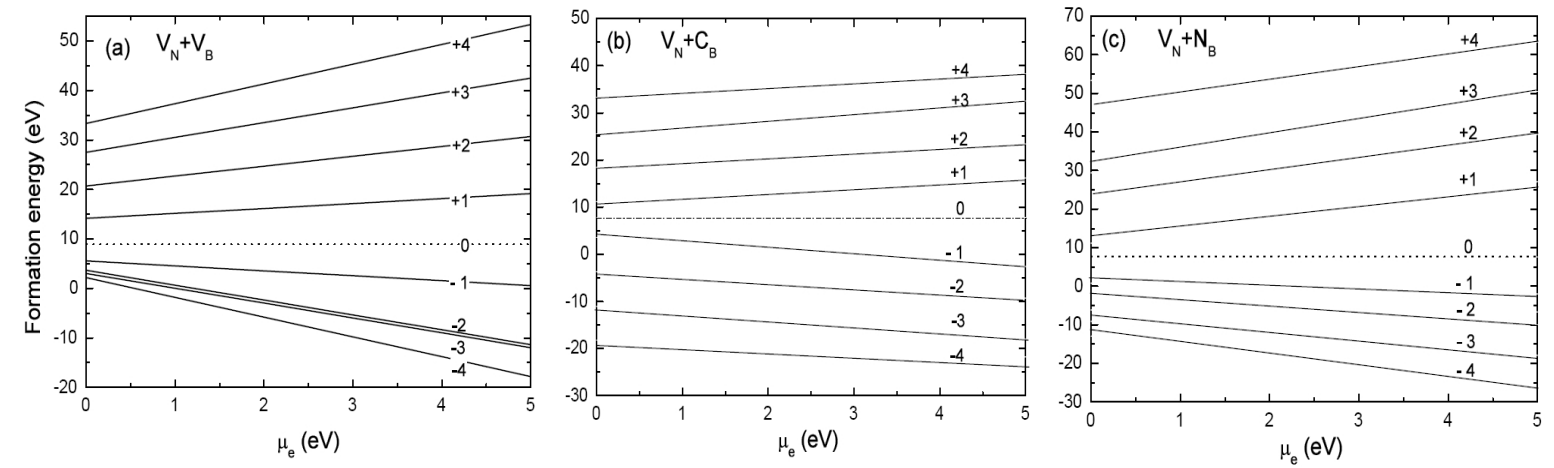

Figure 2. Effect of charge states on the formation energy of nitrogen vacancy complexes for changes in electron chemical potential in nitrogen-rich condition.

Figure 3 presents a summary of the dependence of the formation energy of different neutral defect species on changes in chemical potentials to show boron-rich and nitrogen-rich conditions. Consider that $\mathrm{V}_{\mathrm{N}}+\mathrm{N}_{\mathrm{B}}$ complex and $\mathrm{V}_{\mathrm{B}}$ have the same formation energy within a small interval in the B-rich growth condition, and that formation of carbon-decorated boron and nitrogen vacancies complexes occurs outside this small range (Fig. 3). Because the difference in $E_{f}$ for both defects is less than 0.44 $\mathrm{eV}$ outside this interval, we suggest that it is possible to engineer the $\mathrm{V}_{\mathrm{N}}+\mathrm{N}_{\mathrm{B}}$ complex to give $\mathrm{V}_{\mathrm{B}}$ in suitably B-rich condition. The spontaneous occurrence of this possibility in defect annealing is explored in the following, and we show that an ensemble of transitional structures is obtained through activated nitrogen dynamics. Two unique points exist in the chemical potential where it is possible to form any of $B_{N}, V_{N}$ or $V_{B}+B_{N}$ complex in B-rich growth conditions. It is equally possible to form any of $B_{N}, V_{B}$ or $V_{B}+B_{N}$ defects in the N-rich condition. Because $V_{N}$ is more stable than $V_{B}$, and the substitutional carbon impurity binds more favourably to $V_{N}$ than to $V_{B}$, we conclude that formation of the $\mathrm{V}_{\mathrm{B}}+\mathrm{C}_{\mathrm{N}}$ complex in h-BN is less likely in both growth conditions compared to the $\mathrm{V}_{\mathrm{N}}+\mathrm{C}_{\mathrm{B}}$ complex.

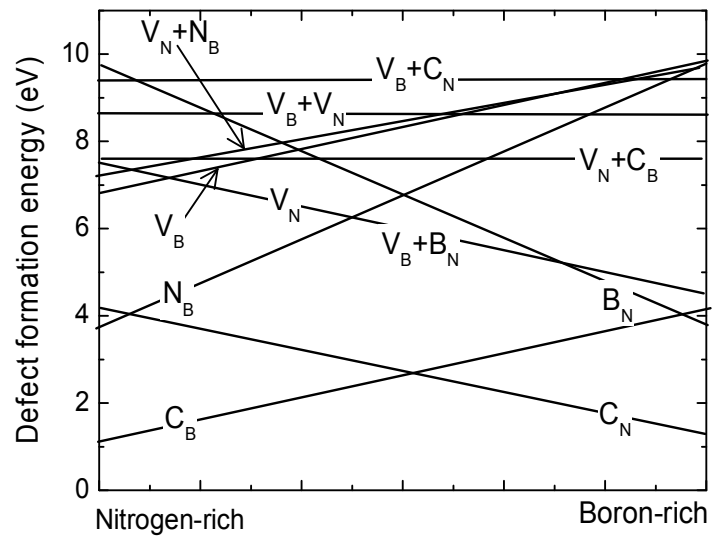

Figure 3. Formation energy of neutral defects as a function of growth condition. 


\subsection{Defect annealing by activated nitrogen dynamics}

The trends in Fig. 3 indicate that formation of point defects is more favourable than formation of complexes, and suggests the possible formation of $V_{B}$ from the $V_{N}+N_{B}$ complex. The $V_{N}+N_{B}$ complex presents its lowest (and highest) formation energy in N-rich (and B-rich) conditions. Its relaxation energy and cohesive energy difference are also higher compared to other nitrogen vacancy complexes. This suggests that nitrogen dynamics is nontrivial. Because the scaling property of the MerminWagner theorem for finite-temperature induced in-plane elastic distortions forbids the spontaneous breaking of the hexagonal symmetry [57], the complexes present only minimal variations in local structure after $50 \mathrm{ps}$ equilibration at $300 \mathrm{~K}$. The minimal variation in structure at finite temperature alludes to the strength of the short-ranged inter-atomic interactions within the layer. These also suggest that any activated migration of nitrogen in the antisite $\left(\mathrm{N}_{B}\right)$ must be important in the process of forming the complexes in both growth environments. To elucidate the structural transitions associated with the activated dynamics of nitrogen, we have investigated the transitional structures by exploring its configurational energy landscape. The transitional configurational space, in this case, is partitioned into saddle points and local minima so that structure optimization on the potential energy surface can map any transitional structure onto the same global energy minimum. The resulting properties of the $\mathrm{V}_{\mathrm{N}}+\mathrm{N}_{\mathrm{B}}$ complex, at a finite temperature, are therefore determined by the ensemble of local minima it can sample, and by the mutual accessibility of local minimum energy points. In finite temperature conditions, the kinetic energy is high, and access to a given local minimum is probabilistic and depends on temperature. Sampling of the energy landscape at $0 \mathrm{~K}$, favors the lowest energy configurations - hence mutual access among finite temperature energy basins is subject to activation.

For each of the transitional structures, we also tested the ground state total energy for convergence to a local minimum at $300 \mathrm{~K}$. In the total energy calculations, the eigenvalues were obtained from the block Davidson and the residual minimization methods to ensure convergence to the same local minimum at the given N-N bond length. The maximum difference in total energy is 6.27 $\mathrm{meV}$ for the equilibrium structure, and less than $8.18 \mathrm{meV}$ for transitional structures with both compressed (short), and stretched (long) N-N bonds respectively in the two methods. The block Davidson minimization scheme consistently yields lower total energies compared to the residual minimization method. As a result, the energy landscape of the minimum energy path discussed below represents lowest total energies for distinct structures in the activated nitrogen dynamics. We therefore investigate the reconstructions that occur when the nitrogen antisite atom migrates from its global minimum position in the $\mathrm{V}_{\mathrm{N}}+\mathrm{N}_{\mathrm{B}}$ complex. We emphasize the changes in local bonding geometries around the complex during its relaxation to lower energy states. The $\mathrm{V}_{\mathrm{N}}+\mathrm{N}_{\mathrm{B}}$ complex and $\mathrm{V}_{\mathrm{B}}$ each contain 24 boron and 25 nitrogen atoms. We show, in the following, that these two defects are energetically in-equivalent. The non-equivalence inhibits a spontaneous transformation from $V_{N}+N_{B}$ $\rightarrow V_{B}$ structure. However, under favourable condition of high local bond strain, the $V_{N}+N_{B}$ complex can become a topologically equivalent defect to the boron vacancy $\mathrm{V}_{\mathrm{B}}$. To illustrate this assertion, we have investigated the minimum energy path associated with such structural transformation. The reaction coordinate is chosen to correspond with the in-plane $\mathrm{N}-\mathrm{N}$ bond length. By varying the N-N bond length from $1.22-2.62 \AA$, we explored the configurational energy landscape prescribed by the $2 \mathrm{D}$ honeycomb structure. Transitional configurations for which the N-N bond length is lower (or higher) than the equilibrium bond length in the equilibrium $\mathrm{V}_{\mathrm{N}}+\mathrm{N}_{\mathrm{B}}$ complex $(1.36 \AA)$ denote configurations with localised compression (or stretching) of the two N-N bonds respectively.

Figure 4 shows the minimum energy path and the unique local structures observed during the simulated nitrogen migration in the $\mathrm{V}_{\mathrm{N}}+\mathrm{N}_{\mathrm{B}}$ complex. An ensemble of transitional structures is found on the energy landscape for both compressed and stretched N-N bonds respectively. The structurally unique local bonding geometries correspond to points A, C, E and F (see Fig 4). The equilibrium structure of the $\mathrm{V}_{\mathrm{N}}+\mathrm{N}_{\mathrm{B}}$ complex is obtained at point $\mathrm{B}$ on the energy landscape with $\mathrm{N}-\mathrm{N}$ bond length of $1.36 \AA$. We note that $\mathrm{V}_{\mathrm{N}}+\mathrm{N}_{\mathrm{B}}$ complex is only able to retain its local bonding structure for $\mathrm{N}-\mathrm{N}$ bonds compressed up to $1.20 \AA$, and for N-N bonds stretched to $1.71 \AA$. The continuous increase in the 
$\mathrm{N}-\mathrm{N}$ bond length beyond $1.88 \AA$ (point $\mathrm{C}$ in Fig. 4) results in the spontaneous annihilation of the two strained N-N bonds. This gives a unique structure akin to Stone-Wales (SW) defect. The reconstructed local structure is an SW(55-66) defect made up of two adjacent pentagon-hexagon pairs, in which a nitrogen atom is bonded to four nearest neighbours. The energy landscape shows that the activation energy of the SW(55-66) defect is $4.994 \mathrm{eV}$ - determined as the energy difference between points $\mathrm{B}$ and $\mathrm{D}$. This energy difference is $6 \mathrm{meV}$ lower than the predicted activation energy of $5.00 \mathrm{eV}$ for formation of an SW(55-77) defect in graphene [58-60]. Fig. 4 indicates that the SW(55-66) defect is comparatively more energetic than the other competing transitional structures. Hence, although the SW(55-66) defect may not form spontaneously due to finite temperature induced structural fluctuations, we predict that its formation via knock-on damage in irradiated monolayers is a distinct possibility [61]. The observation of SW(55-77) defect in graphene monolayers [62-64] is strong indication that possible occurrence of the SW(55-66) defect as a competing structure cannot be overruled.

It is equally possible for spatially well-separated single vacancies to coalesce spontaneously and form the SW(55-77) defect when the in-coming ions are energetic enough to activate the vacancy annealing [65]. A different conformation is obtained at N-N bond length of $2.053 \AA$ (Point E). The local structure is a triad defect formed from two boron atoms located close enough to form the B-B bridge-bond in the B-N-B triangular ring cluster. This triad defect has a low total energy relative to the SW(55-66) defect conformation. We note the resemblance between this triad structure, and the triangle-shaped holes observed in aberration-corrected transmission electron micrographs of asymmetrically-damaged h-BN monolayers [64]. Because monolayer h-BN is more resistant to radiation damage than graphene [66], we do not expect these transitional structures to form spontaneously due to finite-temperature thermal vibrations alone. Their formation can be activated by ion-bombardment, or by irradiation with high-energy electron beams, as long as the threshold for structural damage is exceeded.

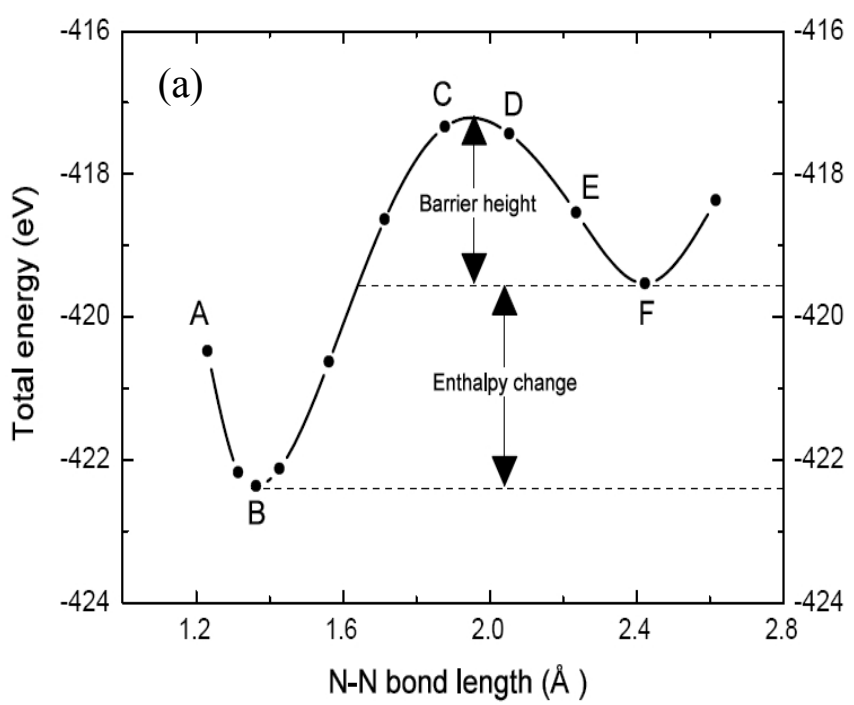

Figure 4. Total energy landscape for nitrogen migration in the $\mathrm{V}_{N}+\mathrm{N}_{B}$ complex (a). The topologically unique local structures observed in the $\mathrm{V}_{\mathrm{N}}+\mathrm{N}_{\mathrm{B}} \rightarrow \mathrm{V}_{\mathrm{B}}$ transformation shows: (b) compressed N-N bonds in the $\mathrm{V}_{\mathrm{N}}+\mathrm{N}_{\mathrm{B}}$ complex (point $\mathrm{A}$ ), (c) two adjacent pentagon-hexagon pairs in an SW(55-66) defect (point $\mathrm{C}$ ), (d) a triad-defect within a $\mathrm{V}_{\mathrm{B}}$-like local structure (point $\mathrm{E}$ ), and (e) non-equilibrium $\mathrm{V}_{\mathrm{B}}$-like defect reconstruction (point $\mathrm{F}$ ). 
The $\mathrm{V}_{\mathrm{N}}+\mathrm{N}_{\mathrm{B}}$ complex relaxes spontaneously into distinct $\mathrm{V}_{\mathrm{B}}$-like local structures beyond point $\mathrm{E}$ on the energy landscape albeit with considerably shortened B-N-B bonds. The local-minimum transitional $\mathrm{V}_{\mathrm{B}}$-like structure is obtained at the saddle point, F. Similarly, the local structure of the highest energy (i.e. most unstable) transitional structure is shown in Fig. 5(a). This is similar to the optimised $V_{B}$ structure - shown in Fig. 5(b). The large difference of $2.84 \mathrm{eV}$, between the total energy at global and local minima (i.e. points $\mathrm{B}$ and F) indicates a non-spontaneous transformation. The migrating nitrogen species has a non-trivial role of mitigating the local bonding transformation. For instance, the nitrogen-mediated bridge bond between the two non-bonded boron atoms of the $\mathrm{V}_{\mathrm{N}}+\mathrm{N}_{\mathrm{B}}$ complex is shortened to $1.368 \AA$, compared to $1.405 \AA$ in the $V_{B}$ structure. The $0.037 \AA$ difference indicates that the N-N bond must carry a $2.63 \%$ strain relative to the boron vacancy, for the $\mathrm{V}_{\mathrm{N}}+\mathrm{N}_{\mathrm{B}}$ complex to transform spontaneously to the $\mathrm{V}_{\mathrm{B}}$ structure. Relative to the global minimum energy $\mathrm{V}_{\mathrm{B}^{-}}$ structure (point $\mathrm{B}$ ), we have observed a continuum of $\mathrm{V}_{\mathrm{B}}$-like local structures, beyond point $\mathrm{F}$, with slightly higher total energies. For this ensemble of $\mathrm{V}_{\mathrm{B}}$-like structures, the B-N-B bridge bonds are significantly shortened and the configurational total energy increases relative to point $F$. The increased total energies indicate increasing instability (due to progressively higher total energies) as the N-B (or B-N) bond strain also increases. Fig. 4 shows that the $V_{N}+N_{B} \rightarrow V_{B}$ conversion is endothermic, with an enthalpy change of $2.84 \mathrm{eV}$. The activation energy (i.e. the potential barrier) for the transformation to occur is $2.38 \mathrm{eV}$. The enthalpies of formation of all the layers with respect to boron and nitrogen atoms at their standard reference state are negative in each case. This is an indication that the migration of nitrogen in the $\mathrm{V}_{\mathrm{N}}+\mathrm{N}_{\mathrm{B}}$ complex can occur spontaneously at finite temperatures if the correct activation energy is supplied to raise the entropy. It is therefore plausible that, at sufficiently high temperatures, the kinetic energy of the migrating nitrogen can cause it to trap into the local minimum (point $\mathrm{F}$ ) to give a metastable $\mathrm{V}_{\mathrm{B}}$ configuration with frozen-in disorder in $\mathrm{B}-\mathrm{N}$ bond.
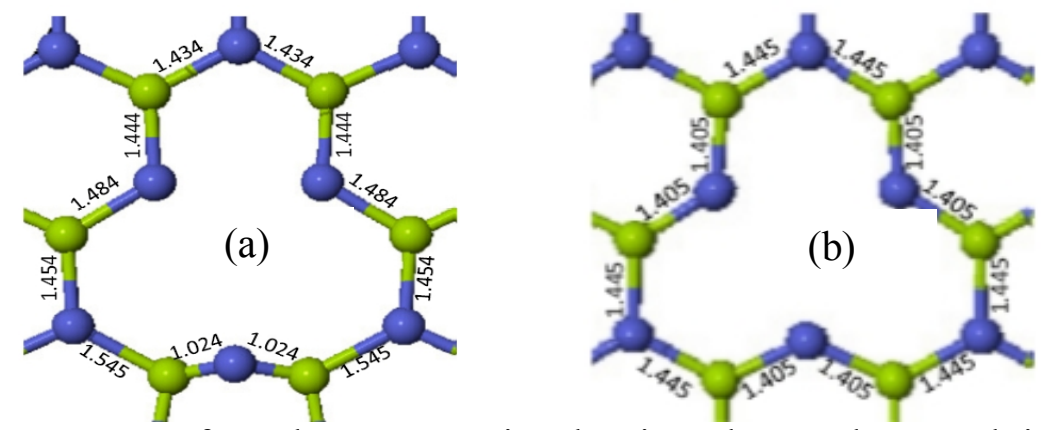

Figure 5. Local structures of two boron vacancies showing: shortened B-N-B bridge bonds in most unstable transitional structure (a), and the equilibrium structure (b).

The above situation contrasts with the spontaneous transformation of the $V_{B}+B_{N}$ complex to $V_{N}$ observed in our previous study [19]. Because the formation energies are lowest when $V_{N}$ forms in $B$ rich condition and highest when $V_{N}+V_{B}$ complex forms in the same condition, the $V_{N}+N_{B}$ complex is unable to trap into metastable configurations beyond a critical point on the energy landscape. We suggest that this critical point corresponds to the unique point in the atomic chemical potential (see Fig. 3) in which the $V_{N}+N_{B}$ and $V_{B}$ defects exhibit the same formation energy. We therefore conclude, vide supra, that the $\mathrm{V}_{\mathrm{N}}$ is energetically more stable than the $\mathrm{V}_{\mathrm{B}}$ in boronitrene irrespective of the conditions of growth. This is consistent with recent experimental observation of nitrogen vacancies in h-BN layers after bombardment with $\mathrm{Ar}^{+}$ions in ultra-high vacuum [67], prior to exposure to air. This interpretation agrees with our calculations, in which the enthalpy of formation of a defective h-BN monolayer with single nitrogen vacancy is $2.28 \mathrm{eV}$ lower than the energy required for forming the same monolayer with a boron vacancy under the most favourable conditions.

Fig. 6 shows buckled structures in defective h-BN networks after equilibration at $3500 \mathrm{~K}$ for both $\mathrm{V}_{\mathrm{B}}+\mathrm{C}_{\mathrm{N}}$ and $\mathrm{V}_{\mathrm{N}}+\mathrm{C}_{\mathrm{B}}$ complexes. Because carbon undergoes trans-vacuum diffusion in both cases, we expect the carbon impurity to originate from a suitable carbon source - preferably graphite. In CVD grown h-BN 
layers, the hyperthermal carbon ions from the decomposing hydrocarbon gas may occupy $\mathrm{B}$ or $\mathrm{N}$ sublattices. Although the melt-quenching procedure used in this study does not describe the mechanism of CVD formation of thin h-BN films [68-71], the melt-quenching kinetics is similar to the pulsed-laser ablation process for producing thin h-BN films [72-77]. Both methods resemble the inelastic thermal spike model [78-81], in which incoming high-energy ions produce localized melting of portions of the high-purity pyrolytic boron nitride target. Subsequent rapid-cooling of film-forming species on small localized regions of the target leads to the ion-assisted film deposition [78-81]. The networks obtained in our simulations represent the non-equilibrium local structures obtained at finite temperatures both from chemical vapour and plasma (or laser) enhanced deposition methods.

The magnitudes of the charge density - with and without carbon in the layer - at $300 \mathrm{~K}$ show that carbon enhances cohesion in the layer. There is also an increased ionicity in $\mathrm{V}_{\mathrm{N}}+\mathrm{C}_{\mathrm{B}}$ complex relative to other nitrogen vacancy complexes, although the magnitude of the difference charge density is higher in $V_{N}+C_{B}$ than in $V_{B}+C_{N}$ complex. The resulting local structure reconstructions suggest that removal of more than two atoms, due to knock-on damage by irradiation, must lead to larger or more complex defect configurations. We suggest that if the number of missing atoms is even, full structure-reconstruction will occur to complete the passivation of dangling bonds. Such open-volume defects would be energetically more favoured than structures with odd number of missing atoms where dangling bonds remain. Although plausible for vacancies and vacancy complexes in h-BN, the presence of the carbon impurity modifies the dynamics of the structure reconstruction significantly. We suggest that if a large number of atoms are instantly removed from a small area of the layer through impact of energetic ions, the reconstruction of the local structure would require significant puckering of the layer to achieve stability because of reduced surface area. An alternative mechanism of local structure reconstruction is through formation of the Haeckelite structure [82], in which equal numbers of pentagons and heptagons are formed in addition to some hexagons [83]. However, this possibility requires specific, preferential, spatial arrangement of vacancies within the layer prior to the structure reconstructions, and it is not clear how such arrangement can be created.
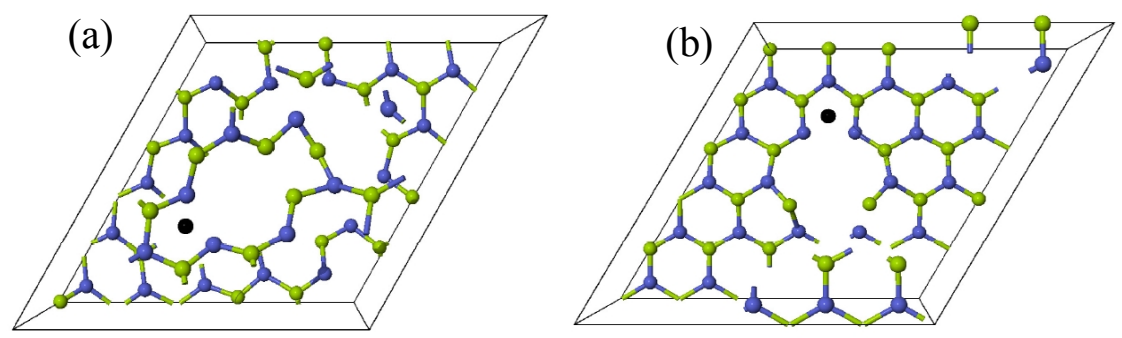

Figure 6. Plan view of the buckled local structures after equilibration at $3500 \mathrm{~K}$ showing: $V_{B}+C_{N}(a)$, and $\mathrm{V}_{\mathrm{N}}+\mathrm{C}_{\mathrm{B}}(\mathrm{b})$. The green and blue balls denote boron and nitrogen atoms, while the black ball denotes a carbon atom. The carbon atom is located within the $15 \AA$ vacuum separations, in each case, and not at the hollow site.

\subsection{Electronic properties}

Figure 7 shows the modified band edges of neutral defect complexes obtained from non-magnetic calculations. Single vacancies $\left(\mathrm{V}_{\mathrm{N}}\right.$ and $\left.\mathrm{V}_{\mathrm{B}}\right)$ and the $\mathrm{V}_{\mathrm{N}}+\mathrm{N}_{\mathrm{B}}$ complex induce a half-filled $s$-like resonant state at the Fermi level [see Figs. 7(a) and (b)]. The band edges in Figs. 7(c) and (d) show that the HOMO and LUMO levels lie below and above the Fermi level in the $\mathrm{V}_{\mathrm{N}}+\mathrm{C}_{\mathrm{B}}$ and $\mathrm{V}_{\mathrm{B}}+\mathrm{V}_{\mathrm{N}}$ complexes. This opens a finite gap between their conduction and valence bands. Relative to the band gap of pristine h-BN monolayer, we find two doubly-occupied, nearly-degenerate defect levels at the top of the valence band at $\left(\varepsilon_{\mathrm{v}}+0.88 \mathrm{eV}\right)$ and $\left(\varepsilon_{\mathrm{v}}+0.90 \mathrm{eV}\right)$ respectively. Two unoccupied levels are also found at the bottom of the conduction band at $\varepsilon_{\mathrm{v}}+0.84 \mathrm{eV}$ and $\varepsilon_{\mathrm{v}}+3.68 \mathrm{eV}$. The inward relaxations of B-N bonds around the centroid of the $\mathrm{V}_{\mathrm{B}}+\mathrm{V}_{\mathrm{N}}$ complex lowers the LUMO level to $0.78 \mathrm{eV}$, leading to a 
reduced band gap of $0.80 \mathrm{eV}$. Within the valence band at point $\mathrm{K}$, two fully-occupied $p$-like levels are found at $-0.11 \mathrm{eV}$ and $-0.23 \mathrm{eV}$. The positions of these two levels relative to the Fermi level shows that the $\mathrm{V}_{\mathrm{B}}+\mathrm{V}_{\mathrm{N}}$ complex suppresses the $s$-resonant state due to the strength of the cohesion in the layer. Nevertheless, as we move away from point $K$, in the directions $\Gamma \rightarrow K$ and $\Gamma \rightarrow M$, the two split defectlevels become degenerate. The two unoccupied defect levels at the bottom of the conduction band suggest that the $\mathrm{V}_{\mathrm{N}}+\mathrm{V}_{\mathrm{B}}$ complex would readily accept electrons promoted from the valence band by either controlled doping or excitation.
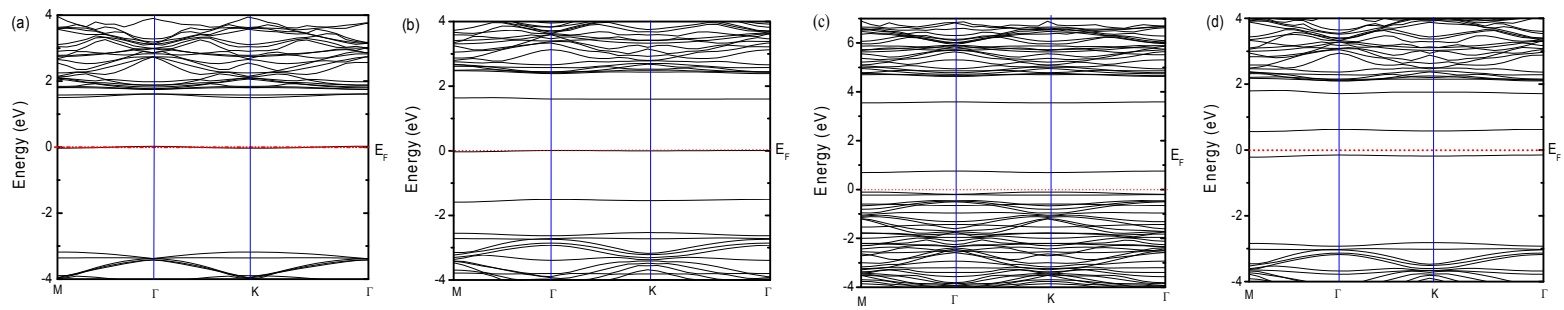

Figure 7. Electronic structures showing perturbed band edges of: $V_{N}(a) ; V_{N}+N_{B}(b) ; V_{N}+V_{B}(c)$; and $\mathrm{V}_{\mathrm{N}}+\mathrm{C}_{\mathrm{B}}(\mathrm{d})$ complexes in boronitrene. The Fermi level corresponds to the zero-energy reference point indicated by dotted horizontal lines at $0 \mathrm{eV}$.

From Fig. 7(c), the band edges within $\pm 4.0 \mathrm{eV}$ shows significant perturbation of the valence and conduction bands in the $\mathrm{V}_{\mathrm{N}}+\mathrm{V}_{\mathrm{B}}$ complex. This perturbation results in the promotion of the halffilled state into the conduction band relative to the $V_{N}$ band edge. Because carbon donates an extra electron to $V_{N}$ in the formation of the $V_{N}+C_{B}$ complex, we suggest that the extra electrons must be spin-paired, resulting in the filled band seen just below the Fermi level in Fig. 7(d). The low-lying defect state at the bottom of the conduction band is the corresponding anti-bonding state. Consider that the cohesive energy difference $\delta$ is only negative in the $\mathrm{V}_{\mathrm{N}}+\mathrm{V}_{\mathrm{B}}$ and $\mathrm{V}_{\mathrm{N}}+\mathrm{C}_{\mathrm{B}}$ complexes (see Table 1). It is plausible that the band gaps between the conduction and valence band are caused by the enhanced structural cohesion in the layer by the complexes. The HOMO-level in both cases lie just below the Fermi level showing fully-filled defect states. The perturbation of the lower conduction band effectively shifts the position of the LUMO-level to low energies. Thus, the calculated band gap is $0.80 \mathrm{eV}$ for direct inter-band transitions in $\mathrm{V}_{\mathrm{N}}+\mathrm{V}_{\mathrm{B}}$, and $0.71 \mathrm{eV}$ for indirect inter-band transitions in $\mathrm{V}_{\mathrm{N}}+\mathrm{C}_{\mathrm{B}}$ complexes. These band gaps are significantly reduced compared to the $5.08 \mathrm{eV}$ and $4.87 \mathrm{eV}$ band gaps expected for direct and indirect inter-band transitions in pristine h-BN [84].

Fig. 8 shows the spin density of states (top panels) and the maximum isosurface of the magnetization density (bottom panels) in three different charge states. The magnetization density is localised in the free-volume of the complex in its +1 charge state, but delocalised on $\mathrm{N}$ atoms along the axis passing through the two non-bonded $\mathrm{N}$ atoms of the complex in the 0 and -1 charged states. The maximum value of the magnetic polarisation density is $4.8 \times 10^{-8},-1.6 \times 10^{-6}$ and $3.4 \times 10^{-2} \mathrm{e} / \AA^{3}$ in the $+1,0$ and -1 charge states, respectively. The negatively-charged spin-polarized state of the $V_{N}+V_{B}$ complex has a magnetic moment of $1.0 \mu_{B}$. This magnetic moment is spontaneous, and can be tuned by charge modulation. Electron (or hole) injection by irradiation and light doping with substitutional impurities can introduce majority carriers that modify the electronic structure [33-35]. The magnetisation density is small in 0 and +1 charge states compared to the -1 state. We ascribe the low magnetization densities to the symmetrical distribution of the spin states in the 0 and +1 charge states. The up and down spin contributions to magnetic moment mutually cancel out because the majority and minority carries have the same density of states. The spin DOS shows that when an extra electron is added to the system $(q=-1)$, the $s$-resonant defect states [see Fig. 7 (a) and (b)] is localised at the Fermi level in the spin up channel. This appears as a sharp peak at $0 \mathrm{eV}$ and gives an overall halfmetallic character to the layer. The maximum isosurface value is 4 orders of magnitude higher than the maximum value of the magnetisation density isosurface in the neutral state. These are consistent with the spontaneous magnetic moments observed in single vacancies $-V_{B}$ and $V_{N}$ - in the h-BN monolayer [17]. In the 0 and +1 charge states, the defect states are localised as band-edge state, thus maintaining 
a small band gap in the electronic structure. The extra electron in the -1 charge state makes it a donor defect, whereas in the +1 charge state, it assumes an acceptor role.
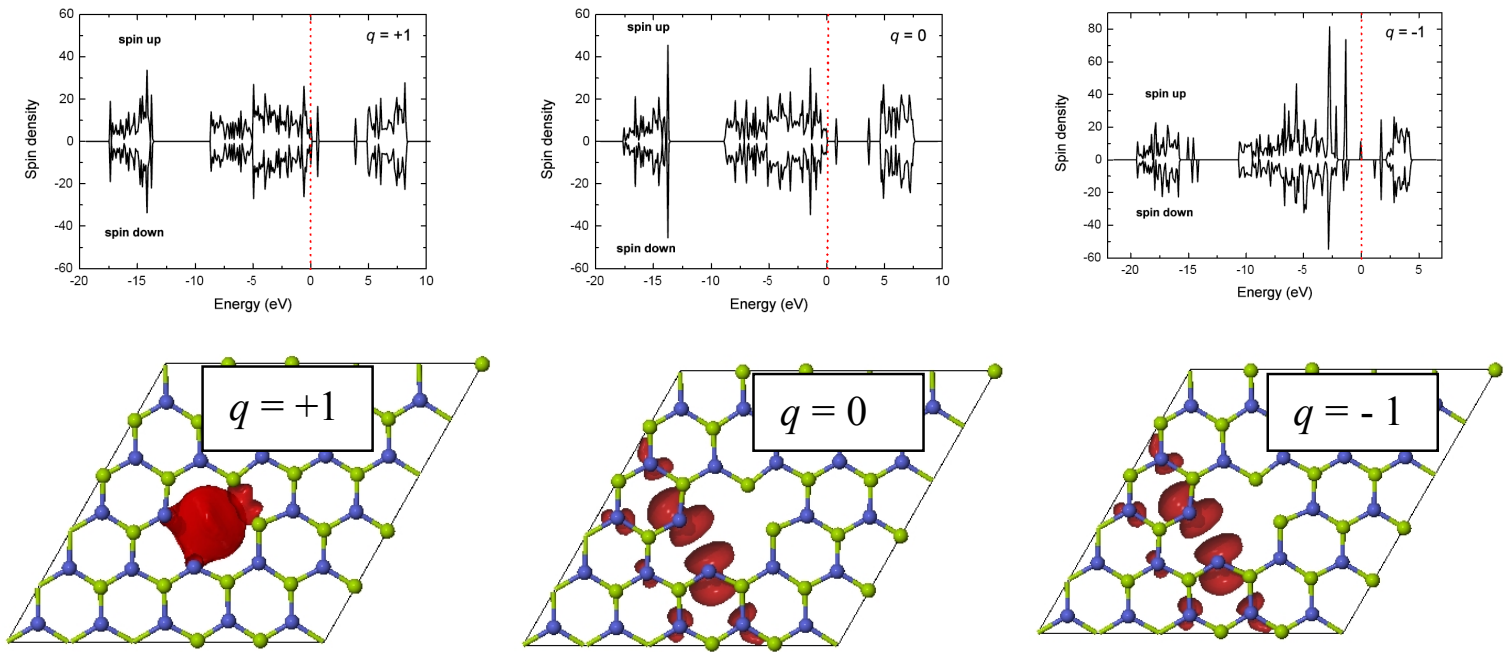

Fig. 8. Total spin density of states (top panels) and magnetic polarisation density (bottom panels) of a $\mathrm{V}_{\mathrm{N}}+\mathrm{V}_{\mathrm{B}}$ complex in the $+1,0$ and -1 charge states. The Fermi level is shown with vertical dotted lines.

\section{Conclusions}

First principles molecular dynamics simulations have been performed to study the nitrogen vacancy complexes in boronitrene. The simulations show that an activated nitrogen dynamics triggers the annihilation of defects in boronitrene. The defect annealing occurs through intermediate processes, which involve the formation of Stone-Wales-type structures in the layer. We use the cohesive energy difference between the defective and pristine boronitrene layers to interpret the modifications of the electronic band structure. In particular, we find that only the complexes that exhibit a negative difference in cohesive energy are able to preserve the band gap between the valence and conduction bands. The divacancy and the carbon-antisite nitrogen-vacancy complexes are identified as viable defect templates for band gap engineering in monolayer boronitrene due to the existence of a finite band gap in their electronic structure. We find that the $s$-resonant defect state at the Fermi level uniquely characterizes the ground state of the nitrogen vacancy complexes, and sets up the spontaneous magnetic moment in monolayer boronitrene that can be tuned by majority carrier injection via irradiation or impurity doping.

\section{Acknowledgements}

We thank Prof. Jannie Pretorius for assistance with the VASP codes. AMU acknowledges financial support from the University of Pretoria under E2020 Project No. 5. NC thanks the University of Pretoria and the National Institute of Theoretical Physics for financial support.

\section{References}

[1] Novoselov K S, Jiang D, Schedin F, Booth T J, Khotkevich T T, Morozov S V and Geim K 2005 Proc. Nat. Acad. Sci. 10210451

[2] Pacilé D, Meyer J C, Girit Ç Ö and Zettl A 2008 Appl. Phys. Lett. 92133107

[3] Alem N, Erni R, Kisielowski C, Rossell M D, Gannett W and Zettl A 2009 Phys. Rev. B 80 155425

[4] Novoselov K S, Geim A K, Morozov S V, Jiang D, Zhang Y, Dubonos S V, Grigorieva I V and Firsov A A 2004 Science 306666 
[5] Novoselov K S, Geim A K, Morozov S V, Jiang D, Zhang Y, Katnelson M I, Dubonos S V, Grigorieva I V and Firsov A A 2005 Nature 438197

[6] Geim A K and Novoselov K S 2007 Nature Mater. 6183

[7] Young A F, Dean C R, Meric I, Sorgenfrei S, Ren H, Watanabe K, Taniguchi T, Hone J, Shepard K L and Kim P 2010 Electronic compressibility of gapped bilayer graphene arXiv:1004.5556

[8] Pacilé D, Meyer J C, Girit Ç Ö and Zettl A 2008 Appl. Phys. Lett. 92133107

[9] Dean C R, Young A F, Meric I, Lee C, Wang L, Sorgenfrei S, Watanabe K, Taniguchi T, Kim P, Shepard K L and Hone J 2010 Nature Nanotech. 5722

[10] Lee C, Li Q, Kalb W, Liu X Z, Berger H, Carpick R W and Hone J 2010 Science 32876

[11] Eisenstein J P and MacDonald A H 2004 Nature 432691

[12] R. M. Ribeiro and N. M. R. Peres, 2011 Phys. Rev. B 83235312

[13] Museur L, Feldbach E and Kanaev A 2008 Phys. Rev. B 78155204

[14] Silly M G, Jaffrennou P, Barjon J, Lauret J S, Ducastelle F, Loiseau A, Obraztsova E, AttalTretout B and Rosencher E 2007 Phys. Rev. B 75085205

[15] Jaffrennou P, Barjon J, Schmid T, Museur L, Kanaev A, Lauret J S, Zhi C Y, Tang C, Bando Y, Golberg D, Attal-Tretout B, Ducastelle F and Loiseau A 2008 Phys. Rev. B 77235422

[16] Yang J, Kim D, Hong J, Qian X, 2010 Surf. Sci. 6041603

[17] Si M S and Xue D S 2007 Phys. Rev. B 75193409

[18] Kan M, Zhou J, Wang Q, Sun Q and Jena P 2011 Phys. Rev. B 84205412

[19] Ngwenya T B, Ukpong A M and Chetty N 2011 Phys. Rev. B 84245425

[20] Makarova T L, Sundqvist B, Höhne R, Esquinazi P, Kopelevich Y, Scharff P, Davydov V A, Kashevarova L S and Rakhmanina A V 2001 Nature 413716

[21] Bandow S, Kokai F, Takahashi K, Yudasaka M and Iijima S 2001 Appl. Phys. A 73281

[22] Esquinazi P, Setzer A, Höhne R, Semmelhack C, Kopelevich Y, Spemann D, Butz T, Kohlstrunk B and Lösche M 2002 Phys. Rev. B 66024429

[23] Wood R A, Lewis M H, Lees M R, Bennington S M, Cain M G and Kitamura N 2002 J. Phys. Condens. Matter 14 L385

[24] Coey J M D, Venkatesan M, Fitzgerald C B, Douvalis A P and Sanders I S 2002 Nature 420156

[25] Meyer J C, Kurasch S, Park H J, Skakalova V, Künzel D, Groß A, Chuvilin A, Algara-Siller G, Roth S, Iwasaki T, Starke U, Smet J H and Kaiser U 2011 Nature Mater. 10209

[26] Wei X, Wang M S, Band Y and Golberg D 2011 ACSNANO 52916

[27] Ci L, Song L, Jin C, Jariwala D, Wu D, Li Y, Srivastava A, Wang Z F, Storr K, Balicas L, Liu F, and Ajayan P M 2010 Nature Mater. 9430

[28] Reddy A L M, Srivastava A, Gowda S R, Gullapalli H, Dubey M and Ajayan P M 2010 ACSNANO 46337

[29] Wang X, Li X, Zhang L, Yoon Y, Weber P K, Wang H, Guo J and Dai H 2009 Science 324768

[30] Han W Q, Wu L, Zhu Y, Watanabe K and Taniguchi T 2008 Appl. Phys. Lett. 93223103

[31] Pruneda J M 2012 Phys. Rev. B 85045422

[32] Kan M, Zhou J, Wang Q, Sun Q and Jena P 2011 Phys. Rev. B 84205412

[33] Wei X, Wang M, Bando Y and Golberg D 2011 ACSNANO 52916

[34] Wei X, Wang M, Bando Y and Golberg D, 2010 J. Am. Chem. Soc. 13213592

[35] Berseneva N, Krasheninnikov A V and Nieminen R M 2011 Phys. Rev. Lett. 107035501

[36] Orellana W and Chacham H 2001 Phys. Rev. B 63125205

[37] Mosuang T E and Lowther J E 2002 Phys. Rev. B 66014112

[38] Schmidt T M, Baierle R J, Piquini P and Fazzio A 2003 Phys. Rev. B 67113407

[39] Liu R F and Cheng C 2007 Phys. Rev. B 76014405

[40] Azevedo S, Kaschny J R, de Castilho C M C and de Brito Mota F 2007 Nanotech. 18, 495707

[41] Azevedo S, Kaschny J R, de Castilho C M C and de Brito Mota F 2009 Eur. Phys. J. B 67507

[42] Hou Z L, Cao M S, Yuan J, Fang X Y and Shi X L 2009 J. Appl. Phys. 105076103

[43] Oba F, Togo A, Tanaka I, Watanabe K and Taniguchi T 2010 Phys. Rev. B 81075125

[44] Kresse G, and Hafner J 1993 Phys. Rev. B 47558 
[45] Kresse G, and Hafner J 1994 Phys. Rev. B 4914251

[46] Kresse G, and Furthmuller J 1996 Phys. Rev. B 5411169

[47] Kresse G, and Furthmuller J 1996 Comput. Mater. Sci. 6, 15

[48] MedeA, Materials design and exploration analysis software, Materials Design, Inc., v. 2.7.2.

[49] Perdew J P, Burke K and Ernzerhof M 1996 Phys. Rev. Lett. 7718

[50] Kresse G and Joubert D 1993 Phys. Rev. B 591758

[51] Blöchl P E 1994 Phys. Rev. B 5017953

[52] Monkhorst H J and Pack J D 1976 Phys. Rev. B 135188

[53] Solozhenko V L and Turkevich V 1999 J. Phys. Chem. B 1032903

[54] Tsay S F and Liu C F 1994 Phys. Lett. A 192374

[55] Zhang S B and Northrup J E 1991 Phys. Rev. Lett. 672339

[56] Şahin H, Cahangirov S, Topsakal M, Bekaroglu E, Akturk E, Senger R. T and Ciraci S 2009 Phys. Rev. B 80155453

[57] Mermin N D and Wagner H, 1996 Phys. Rev. Lett. 171133

[58] Cretu O, Krasheninnikov A V, Rodriguez-Manzo J A, Sun L, Nieminen R M and Banhart F 2010 Phys. Rev. Lett. 105196102

[59] Li L, Reich S and Robertson J 2005 Phys. Rev. B. 72184109

[60] Ma J, Alfé D, Michealides A and Wang E 2009 Phys. Rev. B 80033407

[61] Kotakoski J, Jin C H, Lehtinen O, Suenaga K and Krasheninnikov A V 2010 Phys. Rev. B 82 113404

[62] Kotakoski J, Meyer J C, Kurasch S, Santos-Cottin D, Kaiser U and Krasheninnikov A V 2011 Phys. Rev. B 83245420

[63] Meyer J C, Kisielowski C, Erni R, Rossel M D, Crommie M F and Zettl A 2008 Nano Lett. 8 3582

[64] Meyer J C, Chuvilin A, Algara-Siller G, Biskupek J and Kaiser U 2009 Nano Lett. 92683

[65] Lee G D, Wang C Z, Yoon E, Hwang N M, Kim D Y and Ho K M 2005 Phys. Rev. Lett. 95 205501

[66] Kim J S, Borisenko K B, Nicolosi V and Kirkland A I 2011 ACSNANO 53977

[67] Caretti I, and Jiménez I $2011 \mathrm{~J}$. Appl. Phys. 110023511

[68] Sutter P, Lahiri J, Albrecht P and Sutter E 2011 ACSNANO 57303

[69] Sachdev H, Müller F and Hüfner S, 2011 Angew. Chem. Int. Ed. 503701

[70] Liu Z, Song L, Zhao S, Huang J, Ma L, Zhang J, Lou J and Ajayan P M 2011 Nano Lett. 11 2032

[71] Shi Y, Hamsen C, Jia X, Kim K K, Reina A, Hofmann M, Hsu A L, Zhang K, Li H, Juang Z Y, Dresselhaus M S, Li L J and Kong J 2010 Nano Lett. 104134

[72] Reisse G and Weissmantel S 1999 Appl. Phys. A 69749

[73] Weissmantel S, Reisse G, Keiper B, Weber A, Falke U and Röder M 1998 Appl. Surf. Sci. 127129444

[74] Doll G L, Sell J A, Taylor II C A and Clarke R 1991 Phys. Rev. B 436816

[75] Medlin D L, Friedmann T A, Mirkarimi P B, Rez P, Mills M J and McCarty K F 1994 J. Appl. Phys. 76295

[76] Angleraud B, Girault C, Champeaux C, Garrelie F, Germain C and Catherinot A, 1996 Appl. Surf. Sci. 96-98 117

[77] Pfleging W, Klotzbüchner T, Wesner D A and Kreutz E W 1995 Diamond Relat. Mater. 4370

[78] Toulemonde M, Dufour C, Meftah A and Paumier E 2000 Nucl. Instr. Meth. B 166-167 903

[79] Wang Z G, Dufour C, Paumier E and Toulemonde M 1994 J. Phys. Condens. Matter 66733

[80] Wang Z G, Dufour C, Paumier E and Toulemonde M 1995 J. Phys. Condens. Matter 72525

[81] Dufour C, Audouard A, Beneu F, Dural J, Girard J P, Hairie A, Levalois M, Paumier E and Toulemonde M 1993 J. Phys. Condens. Matter 54573

[82] Terrones H, Terrones M, Hernández E, Grobert N, Charlier J C and Ajayan P M 2000 Phys. Rev. Lett. 841716 
[83] Rocquefelte X, Rignanese G M, Meunier V, Terrones H, Terrones M and Charlier J C 2004 Nano Lett. 4805

[84] Lambrecht W R L and Segal B 1994 Band structure of pure BN Properties of Group-III Nitrides: (EMIS Data Reviews Series), ed J. H. Edgar, (London: INSPEC Publication) p 129. 